\title{
Olfactory fear conditioning induces field potential potentiation in rat olfactory cortex and amygdala
}

\author{
Yannick Sevelinges, Rémi Gervais, Belkacem Messaoudi, Lionel Granjon, \\ and Anne-Marie Mouly ${ }^{1}$
}

Institut des Sciences Cognitives, Unité Mixte de Recherche (UMR) 5015, Centre National de la Recherche Scientifique-Université Lyon 1, and Institut Federatif des Neurosciences (IFR 19), 69675 Bron Cédex, France

\begin{abstract}
The widely used Pavlovian fear-conditioning paradigms used for studying the neurobiology of learning and memory have mainly used auditory cues as conditioned stimuli (CS). The present work assessed the neural network involved in olfactory fear conditioning, using olfactory bulb stimulation-induced field potential signal (EFP) as a marker of plasticity in the olfactory pathway. Training consisted of a single training session including six pairings of an odor CS with a mild foot-shock unconditioned stimulus (US). Twenty-four hours later, the animals were tested for retention of the CS as assessed by the amount of freezing exhibited in the presence of the learned odor. Behavioral data showed that trained animals exhibited a significantly higher level of freezing in response to the CS than control animals. In the same animals, EFPs were recorded in parallel in the anterior piriform cortex (aPC), posterior piriform cortex (pPC), cortical nucleus of the amygdala (CoA), and basolateral nucleus of the amygdala (BLA) following electrical stimulation of the olfactory bulb. Specifically, EFPs recorded before (baseline) and after (during the retention test) training revealed that trained animals exhibited a lasting increase (present before and during presentation of the CS) in EFP amplitude in CoA, which is the first amygdaloid target of olfactory information. In addition, a transient increase was observed in PPC and BLA during presentation of the CS. These data indicate that the olfactory and auditory fear-conditioning neural networks have both similarities and differences, and suggest that the fear-related behaviors in each sensory system may have at least some distinct characteristics.
\end{abstract}

Pavlovian fear conditioning has been one of the most widely used paradigms for studying the neurobiology of learning and memory (for review, see LeDoux 2000; Maren 2001). It consists of pairing an initially neutral stimulus (the conditioned stimulus or CS) with an aversive unconditioned stimulus (US), generally a mild foot-shock. Subsequent re-exposure to the CS elicits a variety of behavioral and physiological responses, such as freezing, considered to reflect a central state of fear. The vast majority of these studies have used auditory CSs, and the corresponding neural network has been well characterized (for review, see LeDoux 2000). The information carried by the auditory CS is relayed to processing areas in the auditory thalamus and proceeds to the auditory association cortex, although both the thalamic and cortical areas send projections to the lateral nucleus of the amygdala, which is a site of CS-US convergence. The lateral nucleus, in turn, projects to the central amygdala, which controls the expression of fear responses through projections to brainstem areas (LeDoux 2000; Maren 2001). Currently, there is general consensus that the amygdala plays a critical role in conditioned fear linking external stimuli to defense responses, as far as auditory or visual stimuli are used for conditioning.

The present study investigated the neural circuit involved in olfactory fear conditioning in rats for two main reasons. First, for rodents, olfaction plays a dominant role in the control of behavior, and previous studies suggest that olfactory learning has unique features concerning acquisition, retention, and extinction (for review, see Slotnick 2001). Second, the olfactory system has unique connections to the amygdala. Indeed, the main olfactory bulb makes dense monosynaptic contacts with nuclei of

\footnotetext{
${ }^{1}$ Corresponding author.

E-mail mouly@isc.cnrs.fr; fax 04-37-91-12-10.

Article published online ahead of print. Article and publication date are at http://www.learnmem.org/cgi/doi/10.1101//m.83604.
}

the corticomedial amygdaloid group, including the nucleus of the lateral olfactory tract, the cortical nucleus of the amygdala (CoA), and the periamygdaloid cortex (Price 1973; McDonald 1998). These observations led Swanson and Petrovich (1998) to suggest that the corticomedial amygdala is an integral component of the olfactory system. These superficial nuclei are a major source of the projections from the amygdala to the hypothalamus (Price et al. 1991). In contrast, the deeper amygdaloid nuclei, including the basolateral nuclear group (BLA), do not receive projections from the olfactory bulb and receive relatively weak projections from the olfactory piriform cortex (Krettek and Price 1978; Ottersen 1982; Luskin and Price 1983). However, they receive fairly dense projections from the corticomedial amygdala (Savander et al. 1996). Taken together, these anatomical data suggest that olfactory information has a unique direct access to the amygdala, with no thalamic relay. Using olfactory cues as CS in fear conditioning will therefore permit the testing of the generality of the current neural models of learning and memory, which are mainly based on auditory stimuli. In addition, our results could provide a particularly relevant model for identifying the relative contribution of sensory cortices and amygdalar nuclei to memory processes.

In parallel to these anatomical considerations, recent behavioral data have shown that olfactory fear conditioning induces robust emotional responses. Otto et al $(1997,2000)$ measuring freezing behavior as an index of learned fear reported that olfactory fear conditioning resulted in robust and long-lasting associations between an olfactory CS and a foot-shock US. Richardson et al (1999), and later, Paschall and Davis (2002) using fear potentiated startle as another measure of learned fear, showed that an odor previously paired with shock was a particularly effective stimulus for potentiating the startle response in rats. Moreover, in a subsequent study, Richardson et al (2002) re- 
ported that extinction of learned fear potentiation of startle occurs more slowly with an olfactory CS than with auditory or visual ones.

Concerning the neural basis of olfactory fear conditioning, very few studies are available in the literature. Olfactory fear conditioning uses the basolateratal amygdala (Sullivan et al. 2000; Moriceau et al. 2004) and Cousens and Otto (1998) showed that pre- and posttraining excitotoxic lesions of the BLA abolished the expression of olfactory fear conditioning in rats. These data were further confirmed by Kilpatrick and Cahill (2003) using reversible inactivation of BLA with tetrodotoxin following paired odorshock presentation in rats. This treatment resulted in a deficit in learning, thus suggesting that BLA plays a role in consolidation of olfactory fear conditioning. Rosenkranz and Grace (2002) performed in vivo intracellular recordings in the lateral nucleus of the BLA during acquisition of an odor fear conditioning under anesthesia, in rats. Their data revealed that repeated pairing of an odor with a foot-shock induced enhanced post-synaptic potential responses in neurons of the BLA, and that this modification resulted from local changes in synaptic efficacy, and was dependant on dopamine. Taken together, these data suggest that BLA plays a major role in olfactory fear conditioning, thus extending to odor cues the previous observations obtained with auditory and visual CS.

Beside the above studies concerning the role of BLA, sparse studies have investigated the potential involvement of other amygala nuclei or of olfactory cortical areas in this learning. Herzog and Otto (1997) showed that anterior perirhinal cortex disrupted fear conditioning to olfactory stimulus. Schettino and Otto (2001) measuring cFos expression related to the acquisition and expression of olfactory fear conditioning, reported that the anterior region of the medial nucleus of the amygdala, as well as ventral perirhinal cortex, could be critically involved in this learning. Using the same technique, Funk and Amir (2000) also showed that presentation of the aversive conditioned odor stimulus induced an enhanced increase in levels of Fos expression in the main and accessory olfactory bulbs and in the anterior olfactory nucleus. However, in behaving animals, no electrophysiological correlates of olfactory fear conditioning have been done. Thus, the major aim of the present study was to investigate whether olfactory fear conditioning induced synaptic changes within the olfactory pathway, mainly focusing on olfactory cortical areas, namely the piriform cortex, and amygdala cortical and basolateral nuclei.

The first part of this study described the main behavioral characteristics of the olfactory fear-conditioning response. The second part consisted of an electrophysiological study carried out during olfactory fear conditioning that took advantage of the well-documented olfactory system and its connections to the amygdala. Evoked field potential signals (EFPs) were induced by electrical stimulation of the olfactory bulb and collected simultaneously in the anterior piriform cortex (aPC), the posterior piriform cortex (pPC), the cortical nucleus of the amygdala (CoA), and the basolateral nucleus of the amygdala (BLA) before and after acquisition of an olfactory fear conditioning.

\section{Experiment 1: Behavioral study}

\section{Results}

Freezing behavior observed during the 8-min CS-test session in animals trained and tested with the Isoamylacetate CS (Group 1) is displayed in Figure 1A. The ANOVA revealed a main effect for the group $\left[F_{(1,10)}=17.463, p=0.002\right]$, the minute $\left[F_{(7,70)}=14.44\right.$, $p<0.001)$, and for group $\times$ minute interaction $\left[F_{(7,70)}=5.084\right.$, $p<0.001]$. Post-hoc tests revealed no statistical difference between groups before the CS odor was introduced (the first $2 \mathrm{~min}$ ).
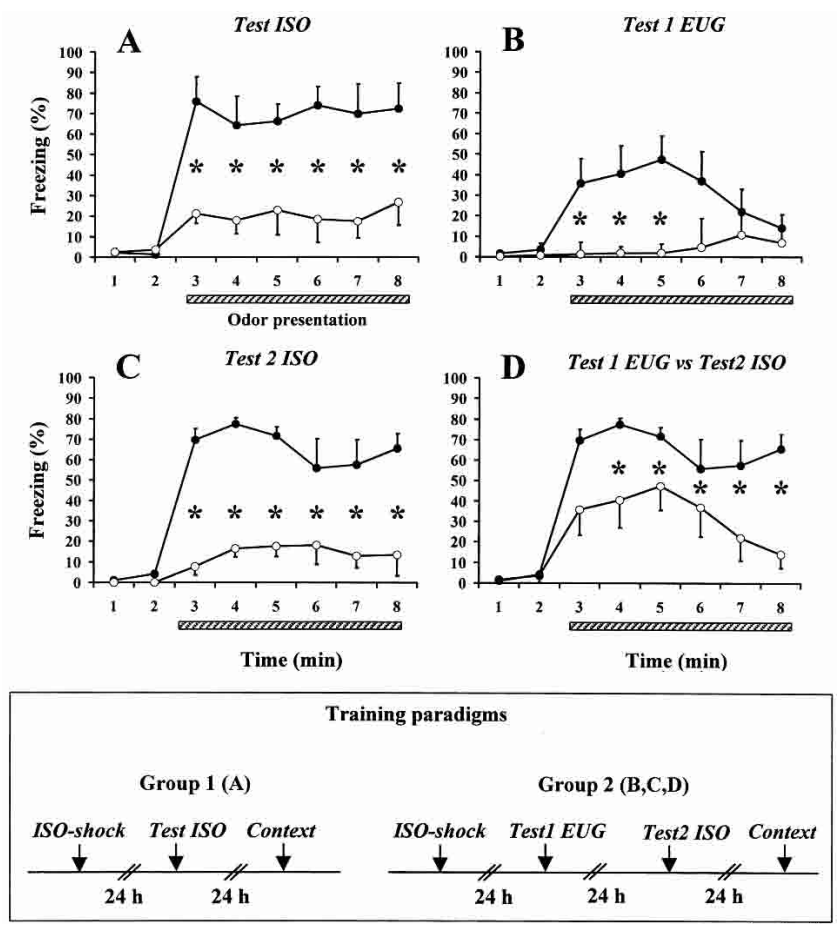

Figure 1. Percentage of freezing (Mean \pm SEM) for each minute of the CS-test session, in the different experimental groups. The bottom of the figure summarizes the training procedures used in the different groups. (A) Group 1, trained animals $(n=6, \bullet)$ and control animals $(n=6,0)$. The animals were trained and tested with Isoamylacetate. (B) Group 2, Test 1 , trained animals $(n=6, \bullet)$ and control animals $(n=6,0)$. The animals were trained with Isoamylacetate and tested with a novel odor, Eugenol. (C) Group 2, Test 2, trained animals $(n=6, \bullet)$ and control animals $(n=6$, O). Twenty-four hours after Test 1 , the animals were tested with Isoamylacetate CS. (D) Group 2, trained animals, Test 1 vs. Test 2. Comparison of the freezing response to the Isoamylacetate $\operatorname{CS}(n=6, \bullet)$ and to Eugenol $(n=6,0)$. (*) Significant difference between the two sessions $(p<0.05)$.

However, from minutes $3-8$, i.e., when the Isoamylacetate CS was presented, trained animals exhibited significantly higher levels of freezing than control animals.

To assess odor specificity, another group of animals (Group 2) was trained with Isoamylacetate, but later tested with a novel odor (Eugenol), and $24 \mathrm{~h}$ later with the conditioned odor (Isoamylacetate). Results from the novel Eugenol odor test showed that trained rats compared with control showed no freezing prior to the introduction of the novel odor, but significantly more freezing for the first few minutes of the odor presentation (Fig. $1 B)$. Specifically, the ANOVA revealed a significant difference for the factors group $\left[F_{(1,10)}=14.592, p=0.003\right]$, minute $\left[F_{(7,70)}=3.379, \mathrm{p}=0.004\right]$, and for group $\times$ minute interaction $\left[F_{(7,70)}=3.302, \mathrm{p}=0.004\right]$. Post-hoc tests revealed no difference between the two groups during the first $2 \mathrm{~min}$, i.e., before introduction of Eugenol. At minute 3, i.e., when Eugenol was presented, trained animals showed a significantly higher percentage of freezing than control animals $(p=0.019)$. This difference remained significant until minute $5(p<0.05)$, after which the two groups no longer differed statistically. These data suggest there was some generalization from the conditioned odor to the novel odor.

Animals were then tested with the odor that was present during conditioning, i.e., Isoamylacetate. (Fig. 1C). The ANOVA revealed a significant effect for the factors group $\left[F_{(1,10)}=53.29\right.$, $p<0.001]$, minute $\left[F_{(7,70)}=18.44, p<0.001\right]$, and for group $\times$ minute interaction $\left[F_{(7,70)}=7.88, p<0.001\right]$. Post-hoc comparisons showed that there was no significant difference be- 
tween trained and control animals during the first 2 min of the session, but that from minutes $3-8$, i.e., as long as the conditioned odor Isoamylacetate was present, trained animals showed a significantly higher percentage of freezing than control animals $(p<0.05)$. We further compared the freezing behavior displayed by trained animals during the two test sessions (Eugenol vs. Isoamylacetate, Fig. 1D). Wilcoxon comparisons revealed that, from minutes $4-8$, the percentage of freezing elicited in response to the conditioned odor Isoamylacetate was significantly higher than that observed in response to novel Eugenol odor.

Contextual conditioning was assessed in Groups 1 and 2, during a specific context testing session as displayed in Table 1 . Specifically, in Group 1, the ANOVA revealed no effect for the factors group $\left[F_{(1,10)}=0.266, p=0.617\right]$ nor for group $\times$ minute interaction $\left[F_{(5,50)}=0.132, p=0.984\right]$. Similarly, in Group 2, trained and control animals were not significantly different from one another. Indeed, ANOVA revealed no effect for the factors group $\left[F_{(1,10)}=3.358, p=0.097\right]$ nor for group $\times$ minute interaction $\left[F_{(4,40)}=0.976, p=0.431\right]$. Therefore, trained animals in Groups 1 and 2 did not learn contextual cues.

\section{Discussion}

Previous studies have shown that animals trained in a classical fear-conditioning paradigm exhibit freezing responses when reexposed to either the context in which conditioning occurred, or to the CS in a separate context. In our learning paradigm, animals trained and tested to the CS odor (Group 1) exhibited robust freezing responses when re-exposed to the learned CS Isoamylacetate. The conditioned fear response lasted the entire duration of the test, thus confirming the data previously reported by Otto et al $(1997,2000)$ that suggests olfactory cues show long retention rates.

However, in our odor specificity test (Group 2), some freezing was elicited by presentation of a novel odor. Specifically, the data showed that Eugenol induced a significant freezing response in animals trained with Isoamylacetate as a CS. However, the amplitude of this freezing response was lower and its duration shorter than those obtained in the presence of the CS. Isoamylacetate and Eugenol belong to two different chemical families (Ester for the former and alcohol for the latter) and are qualitatively very different. Therefore, freezing obtained in response to Eugenol can hardly be ascribed to similarities between the two odors. It can rather be explained by a relative generalization of the conditioned fear response to any odorant presented in the experimental situation.

No contextual conditioning was detected in our animals. Specifically, no freezing response was detected in trained animals during the first 2 min of the CS-test session, before introduction of the CS. Measuring animals' behavior before introduction of the CS in a test cage different from the conditioning context gives information about generalization phenomenon (freezing when no CS is present, and outside the conditioning context). Therefore, it can be concluded that in the present study, the trained animals did not generalize the conditioned response to the contextual cues of the CS-testing cage.

Additionally, no freezing response was observed when trained animals were re-exposed to the training context. There-

Table 1. Percentage of freezing (mean \pm SEM) for each minute of the context-test session, in the different experimental groups

\begin{tabular}{lccrrrr}
\hline Minutes & $\mathbf{1}$ & $\mathbf{2}$ & $\mathbf{3}$ & $\mathbf{4}$ & $\mathbf{5}$ & $\mathbf{6}$ \\
\hline Trained group 1 & $1 \pm 1$ & $2 \pm 1$ & $11 \pm 6$ & $7 \pm 3$ & $13 \pm 5$ & $16 \pm 9$ \\
Control group 1 & $1 \pm 1$ & $0.5 \pm 0.3$ & $10 \pm 3$ & $4 \pm 2$ & $13 \pm 5$ & $10 \pm 3$ \\
Trained group 2 & $0 \pm 0$ & $6 \pm 5$ & $8 \pm 4$ & $26 \pm 7$ & $13 \pm 5$ & $16 \pm 8$ \\
Control group 2 & $0 \pm 0$ & $0 \pm 0$ & $1 \pm 1$ & $8 \pm 5$ & $5 \pm 2$ & $9 \pm 4$ \\
\hline
\end{tabular}

fore, in our experimental conditions, animals did not associate the contextual cues of the training cage to the foot-shock. In a recent study, Baldi et al (2004) examined the relationship between foot-shock intensity and the freezing response to both CS cue and the context. They reported that both cue and contextual conditioning was found with a $0.5-\mathrm{mA}$ intensity foot shock, although only cue conditioning was found for the milder $0.3-\mathrm{mA}$ foot-shock intensity. In the present study, we used $0.4 \mathrm{~mA}$ footshock intensity, and our results suggest this intensity of footshock is also too mild to support contextual conditioning.

Taken together, the behavioral data show that our experimental animals developed odor-shock cue learning with no contextual learning. Although not strictly specific, the conditioned response appeared, however, to be stronger for the odorant used as a CS during training.

\section{Experiment 2: Electrophysiological study}

\section{Results}

\section{Behavioral data}

The animals used for EFPs were tested for their freezing response during CS and context test sessions. As shown in Table 2, behavioral results were similar to the results of Experiment 1, although baseline immobility ratings were higher across both the trained and control animals, perhaps due to the presence of the recording cables. However, trained animals showed significantly more odor-induced freezing behavior than control animals with no contextual conditioning. Specifically, the ANOVA revealed a main effect for minute $\left[F_{(7,105)}=11.117, p<0.001\right]$ and for group $\times$ minute interaction $\left[F_{(7,105)}=2.66, p=0.014\right]$. Post-hoc tests revealed no significant difference between groups for the first $2 \mathrm{~min}$, i.e., before the introduction of the CS. At minute 3 , i.e., when Isoamylacetate was introduced, animals in the trained group presented a significantly higher level of freezing than control animals. This difference remained significant at minute 4, after which control and trained animals did not differ anymore. Further, intragroup comparisons showed that in trained animals, but not in control animals, freezing behavior exhibited during minutes 3-8 was significantly higher than that observed during the first 2 min of the session ( $p<0.01$ for all the comparisons).

Concerning context-test, the ANOVA revealed no effect for the factors group $\left[F_{(1,14)}=0.002, p=0.969\right]$ nor for group $\times$ minute interaction $\left[F_{(5,70)}=0.176, p=0.971\right]$.

\section{Electrophysiological data}

As illustrated in Figure 2, EFPs were collected at each recording site during the $2 \mathrm{~min}$ prior to CS odor introduction (pre-odor period) and the 6 min during odor (odor-period), and compared with baseline preconditioning signals. The ANOVA revealed the following differences: concerning the factor group, a significant effect was obtained in CoA $\left[F_{(1,14)}=4.67, p=0.048\right]$ and BLA $\left[F_{(1,14)}=7.10, p=0.018\right]$, and a tendency in pPC $\left[F_{(1,15)}=3.85\right.$, $p=0.069]$. Concerning the factor period, a significant effect was obtained in pPC $\left[F_{(1,15)}=9.34, p=0.008\right]$ and $\operatorname{CoA}\left[F_{(1,14)}=7.12\right.$, $p=0.018]$, and a tendency in BLA $\left[F_{(1,14)}=4.03, p=0.060\right]$. No significant effect of either factor was observed in aPC.

Wilcoxon comparisons carried out in the trained group revealed that during the pre-odor period, i.e., the first 2 min of the session when the animal was in the test cage, but received no odor stimulation, there was a significant increase in the amplitude of EFP sig- 
Table 2. Percentage of freezing (mean \pm SEM) for each minute of the CS-test and context-test, in trained $(n=9)$ and control $(n=8)$ implanted animals

\begin{tabular}{lcccccccc}
\hline \multicolumn{7}{c}{ CS-test } \\
\hline Minutes & $\mathbf{1}$ & $\mathbf{2}$ & $\mathbf{3}$ & $\mathbf{4}$ & $\mathbf{5}$ & $\mathbf{6}$ & $\mathbf{7}$ & $\mathbf{8}$ \\
\hline Trained & $18 \pm 9$ & $26 \pm 8$ & $84 \pm 4^{\mathrm{a}, \mathrm{b}}$ & $88 \pm 4^{\mathrm{a}, \mathrm{b}}$ & $82 \pm 7^{\mathrm{b}}$ & $78 \pm 6^{\mathrm{b}}$ & $76 \pm 8^{\mathrm{b}}$ & $75 \pm 1^{\mathrm{b}, \mathrm{a}}$ \\
Control & $35 \pm 14$ & $33 \pm 11$ & $43 \pm 12$ & $60 \pm 12$ & $60 \pm 10$ & $55 \pm 15$ & $61 \pm 12$ & $63 \pm 12$ \\
\hline \multicolumn{7}{c}{ Context-test } \\
\hline Minutes & 1 & 2 & 3 & 4 & 5 & \\
\hline Trained & $8 \pm 5$ & $30 \pm 8$ & $30 \pm 9$ & $30 \pm 12$ & $53 \pm 9$ & $58 \pm 14$ \\
Control & $15 \pm 6$ & $26 \pm 11$ & $35 \pm 11$ & $41 \pm 11$ & $54 \pm 15$ & $58 \pm 12$ \\
\hline
\end{tabular}

aBetween group comparisons, significant difference between trained and control animals $(p<0.05)$.

${ }^{b}$ Within group comparisons, significant difference compared with the first 2 min in the CS-test session $(p<0.05)$.

nal collected in the CoA $(p=0.025)$ compared with baseline. No significant effect was observed for the other recording sites. Concerning the odor-period, i.e., the 6 min during which the learned odor was presented, a significant increase in signal amplitude compared with baseline was observed in CoA (0.012) and BLA $(p=0.038)$. Comparisons between pre-odor and odor periods additionally showed that introduction of the learned odor induced significant increases in two recording sites as follows: pPC $(p=0.021)$ and $\mathrm{CoA}(p=0.05)$.

In the control group during the pre-odor period, a tendency to decrease was observed in all the recording sites, but it did not reach a significant level. During the odor period, no significant variation in EFP amplitude compared with baseline was observed.

For each recording site, mean EFP signals obtained for each single minute of the CS-test session were then compared with baseline signals in order to test for the temporal dynamics of the observed modifications in a given site throughout the session. The data are represented in Figure 3. The ANOVA revealed a main effect of group $\left[F_{(1,54)}=16.878, p<0.001\right]$ and of minute $\left[F_{(7,378)}=6.414, p<0.001\right]$. Further Wilcoxon comparisons revealed that in CoA of trained animals, the mean per minute EFPs amplitudes were significantly greater than baseline from minutes $1-8(p<0.05$, except for minute 2 , for which only a statistical tendency was observed; $p=0.069$ ). In addition, the enhancement described above in CoA between pre-odor and odor periods appeared to peak at minutes 3 and 7 . In the BLA, a tendency to increase was observed during minutes 1 and 2 , but it did not reach a significant level. The further enhancement observed during the odor period was significant for minutes $3,4,5$, and 7 $(p \leq 0.05)$. Finally, the enhancement observed in pPC during the odor period compared with the pre-odor period appeared to peak at minutes 3 and 6 .

\section{Discussion}

\section{Behavioral data}

Trained and control animals presented the same level of freezing during the first two minutes of the CS-test session, i.e., before introduction of the odor. Following introduction of the conditioned odor, trained animals exhibited significantly higher levels of freezing than control animals. This difference remained significant until minute 4 , after which trained and control animals presented the same level of freezing. Therefore, compared to data from Experiment 1, which showed a significant difference between trained and control animals from minutes $3-8$, the differ- ence between trained and controlimplanted animals vanished rapidly. However, it must be noted that implanted animals in both trained and control groups presented basal levels of nonassociative freezing significantly higher than those observed in nonimplanted animals in Experiment 1. This difference might be due to the fact that surgery has rendered implanted animals more anxious, or that recording cables connecting the animals to the apparatus disrupted animals' spontaneous behavior. The high level of basal freezing in control-implanted rats could have thus masked the conditioned freezing response in trainedimplanted animals. Nevertheless, intragroup comparisons revealed that, unlike control animals, the freezing response observed in trained animals during each of the $6 \mathrm{~min}$ of CS-odor presentation was significantly higher than that displayed before odor introduction.

\section{Electrophysiological data}

Cellular origins of the recorded signals

The EFP observed in piriform cortex in response to lateral olfactory tract stimulation has been well described in the literature (Haberly 1973; Ketchum and Haberly 1993) and mainly consists of a large surface-negative, deep-positive wave thought to be generated by a monosynaptic excitatory postsynaptic potential in pyramidal cells. The EFP recorded in cortical amygdala has been much less studied (but, see McNamara et al. 2004), however, this structure presents many anatomical similarities with the piriform cortex regarding its cellular components and laminar organization. It can thus be assumed that the EFP components recorded in this structure share the same characteristics as those recorded in the piriform cortex. Therefore, in aPC, pPC, and CoA, an increase in EFP peak amplitude can be interpreted as an enhancement of the excitatory response of the population of neurons
A

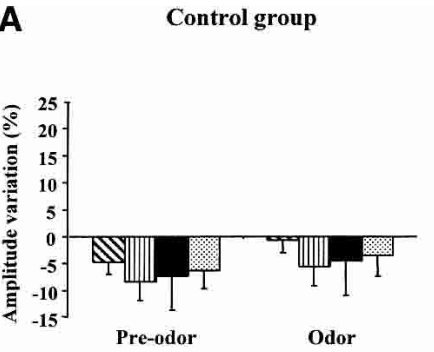

వaPC MpPC
B Trained group

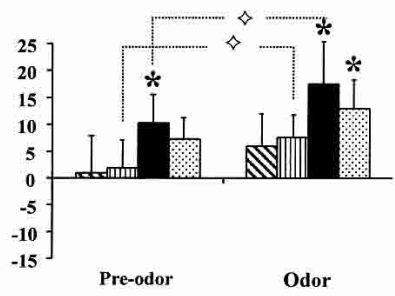

Figure 2. Mean ( \pm SEM) variation in EFPs amplitude measured in control $(A, n=8)$ and trained $(B, n=9)$ animals, in the four recording sites, during the CS-test session. Changes are expressed in percentage of variations compared with the amplitude of the baseline obtained before conditioning. EFPs collected during the first $2 \mathrm{~min}$ of the session (Pre-odor period) and during the 6 min of CS odor presentation (Odor period) were pooled separately. (aPC) Anterior piriform cortex; ( $\mathrm{pPC}$ ) posterior piriform cortex; (COA) cortical nucleus of the amygdala; (BLA) basolateral amygdala. $(\diamond)$ Significant difference between Pre-odor and Odor periods $(p<0.05)$. (*) Significant difference compared with baseline signals $(p<0.05)$. 

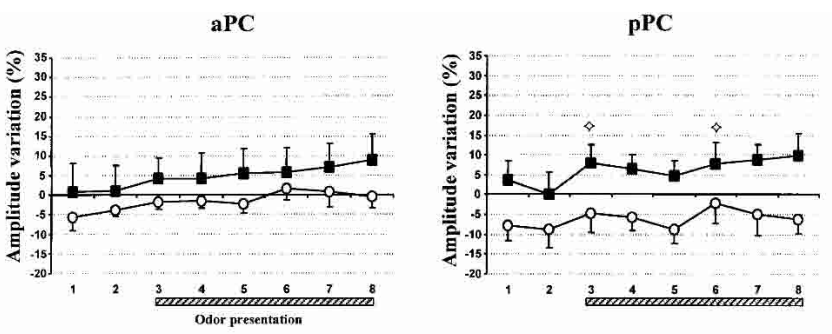

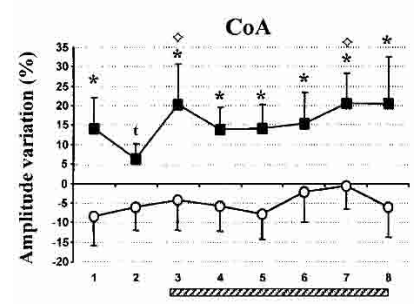

Time (min)

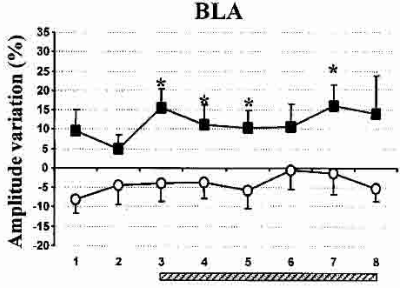

Time (min)
-1- Trained group -O-Control group

Figure 3. Mean $( \pm$ SEM) variation in EFPs amplitude of signals collected in the four recording sites, in trained $(n=9)$ and control $(n=8)$ animals, during the 8-min CS-test session. The EFPs collected during each minute of the session were averaged and analyzed separately. Changes are expressed as percent variations from the baseline signals collected before training. ( $\mathrm{aPC}$ ) Anterior piriform cortex; ( $\mathrm{PPC}$ ) posterior piriform cortex; (CoA) cortical nucleus of the amygdala; (BLA) basolateral amygdala. $\left(^{*}\right)$ Significant difference compared with baseline signals $(p<0.05)$. $(\diamond)$ Significant difference with at least 1 of the first $2 \min (p<0.05)$.

sampled by the electrode. Concerning EFP recorded in basolateral amygdala, the interpretation is more subject to caution since there are no direct projections from the olfactory bulb to the BLA. Therefore, the main component of the signal is at least disynaptic. An increase in BLA signal peak amplitude can thus be interpreted as resulting from enhanced transmission within BLA or from a distal increase in a structure sending projections to the BLA.

\section{Advantages and limitations of the technical approach}

We chose to use electrically induced EFPs instead of olfactoryinduced EFPs for two main reasons. First, an odor unlike a tone (Rogan et al. 1997; Tang et al. 2001, 2003) hardly induces measurable EFPs that can be studied in freely moving animals in the course of a fear-conditioning paradigm. Second, using electrical stimulation to induce EFPs allowed us to investigate whether learning-induced changes could be detected in the absence of CS, and of the associated fear response. This advantage is of importance in order to differentiate changes linked to the processing of the learned CS that are concomitant with the expression of conditioned fear from changes outlasting the presentation of the CS, which can therefore be dissociated from the behavioral response.

The EFP signals recorded in the present study represent the synchronous postsynaptic activation of a large number of cells in each structure. Increase in EFP amplitude can thus be interpreted as a global enhancement of the response of this population. In the current view of memory (Martin and Morris 2002), these learning-related lasting changes could represent the substrate of the memory trace of the learned odor. Although the size of the observed changes (10\%-20\%) could be considered as small compared with that reported in studies using natural auditory stimuli (100\%-150\%; Rogan et al. 1997; Tang et al. 2003), it is, however, in the same range as that obtained in experiments using electrically induced EFPs (Doyère et al. 1995; Herry et al. 1999; Desmedt et al. 2003). Interestingly, in the olfactory system, Wilson (1998a) compared the changes obtained in the piriform cortex of anesthetized rats following habituation to different odors (among which was Isoamylacetate), on the synaptic response evoked by either the odor or the electrical stimulation of the lateral olfactory tract. The investigator reported that the changes observed on odor-evoked signals $(60 \%-70 \%)$ were far greater than those seen on electrically induced signals (15\%-20\%). The investigator argued that electrical stimulation of the olfactory bulb is nonspecific compared with the probable effects of odor stimulation, and has probably activated both habituated and nonhabituated synapses. Similarly, in the present experiment, both potentiated and nonpotentiated synapses might have been activated by electrical stimulation of the olfactory bulb, thus explaining the relatively low size of the observed changes.

\section{Summary of the observed changes in EFP signals}

The present study was designed to investigate whether synaptic changes could be detected at different levels of the olfactory pathways following olfactory fear conditioning in awakebehaving animals. The data showed that olfactory fear conditioning was accompanied by (1) a lasting increase in CoA, i.e., an increase observed in presence as well in absence of stimulus presentation, (2) a transient increase in BLA, i.e., an increase observed in presence of the CS, (3) a facilitation of the potentiation by odor presentation, in pPC and CoA.

\section{General interpretation of the observed potentiations}

All of the potentiation phenomenons described in trained animals were absent in control animals. Therefore, they cannot be ascribed to the nonspecific effects of repeated daily electrical stimulation of the olfactory bulb.

Concerning the transient increases, i.e., those observed in the presence of the CS odor, whereas the animals exhibited a robust conditioned freezing response, it could be argued that these changes are a consequence of nonspecific effects of the behavioral response, like modulation of brain temperature by freezing at the time of EFPs sampling (Moser et al. 1993). Although we cannot entirely discard this possibility, two kinds of arguments allow us to suggest that it is unlikely. First, controlimplanted animals presented a high level of basal nonassociative freezing, yet their EFP signals did not vary significantly throughout the CS-test session, even presenting a tendency toward a global decrease. Second, in a recent experiment, Goosens et al. (2003) studying auditory-evoked spike firing in the lateral amygdala following fear conditioning found that the expression of conditional freezing was neither necessary nor sufficient for the expression of conditional increases in CS-evoked spike firing.

The lasting increases, i.e., the increases observed during the first 2 min of the session, cannot be ascribed to the expression of a fear state. Indeed, this increase was observed when the CS odor was absent, whereas the animals exhibited a freezing response identical to that observed in control animals. It could be argued that the enhancement observed before introduction of the CS is the consequence of lasting modifications sustaining learning of contextual cues. However, the behavioral data from the contexttest revealed that trained animals did not exhibit freezing when re-exposed to the training context, thus confirming the data obtained in Experiment 1. Therefore, it can be suggested that this potentiation is a lasting consequence of the associative CS-US learning. Whether this potentiation is also present when the animal is in its home cage or is expressed only when the rat is introduced in the test cage would deserve further investigations.

\section{Transient potentiation in $p P C$}

The transient increase observed in pPC during odor presentation in trained animals could be interpreted as an enhancement of 
activity due to sensorial processing of the odor. However, in control animals, no increase was induced during odor presentation. The increase obtained in trained animals can therefore be ascribed to the effect of learning. As this facilitation was only observed in presence of the learned odor, its expression could be under the dependence of neuromodulators involved in this task. In particular, it is well known that locus coeruleus is activated in response to meaningful stimulus (Sara and Segal 1991), and that noradrenaline plays an important role in fear conditioning (for review, see McGaugh 2004). The piriform cortex receives noradrenergic input from the locus coeruleus (Datiche and Cattarelli 1996), and noradrenaline has been shown to modulate synaptic transmission in the piriform cortex (Hasselmo et al.1997). In a recent study carried out on anaesthetized rats (Bouret and Sara 2002), locus coeruleus stimulation was shown to enhance piriform cortex cells' responses to odors. Interestingly, the investigators reported that the proportion of stimulation-induced increase in response magnitude was higher in pPC than in aPC. In our study, locus coeruleus activation in response to the learned stressful odor could be responsible for the enhancement observed in pPC. No increase was observed in aPC in trained animals. These data bring further support to accumulating evidence that aPC and pPC seem to play different roles in memory processes (Litaudon et al. 1997, 2003; Chabaud et al. 1999, 2000; Mouly et al. 2001, Mouly and Gervais 2002). In particular, aPC would be mainly involved in sensory processes, and simple forms of shortterm memory like habituation (Wilson 1998b, 2000) or perceptual learning (Wilson 2003), whereas pPC would be involved in the learning and recall of associations between odorants and information from other sensory modalities (Haberly 2001).

\section{Transient potentiation in BLA}

The increase in BLA was only significant in the presence of the CS odor. However, it must be noted that a tendency to increase was also observed during the first 2 min of the session, suggesting that some substhreshold lasting modifications might also have occurred in the BLA. The changes were nevertheless qualified as transient with regard to statistical significativity.

As for the pPC, this transient enhancement could be modulated by the release of neuromodulators upon arrival of the stressful odor. Indeed, many experiments indicate that stress hormones released during emotionally arousing experiences play a critical role in consolidating lasting memories, and that amygdala is the critical site of their action (for review, see McGaugh and Roozendaal 2002). In addition, epinephrine and glucocorticoids' effects are intimately linked to noradrenergic activation in the BLA. Noradrenaline release in the amygdala could enable long-term memory consolidation in other brain regions (McGaugh 2002, 2004). Concerning olfactory fear conditioning, a few studies have already shown that BLA is involved. Indeed, Sullivan et al. (2000) and Sullivan (2001) reported that before the age of $10 \mathrm{~d}$, rat pups have difficulties in learning odor-shock aversions and attributed this deficit to the lack of a functional amygadala at this early age of life. In adult rats, Cousens and Otto (1998) showed that pre- and post-training exitotoxic lesions of the BLA abolished the expression of olfactory fear conditioning. Recently, Kilpatrick and Cahill (2003) showed that reversible inactivation of BLA following paired odor-shock presentation in rats resulted in a deficit in learning, suggesting that BLA is involved in consolidation of olfactory fear conditioning. Rosenkranz and Grace (2002) performing in vivo intracellular recordings in the BLA during acquisition of an odor fear conditioning under anesthesia in rats, demonstrated that repeated pairing of an odor with a foot-shock induced enhanced post-synaptic potential responses to the odor in neurons of the BLA, and that this modification resulted from local changes in synaptic efficacy, and was dependant on dopamine. These changes could constitute the basis of the changes observed in the present study in trained animals in the presence of the learned odor.

\section{Lasting potentiation in $\mathrm{CoA}$}

The enhancement observed in CoA is expressed before introduction of the CS-odor, and is further increased in the presence of the learned odor. Interestingly, the increase during odor is statistically significant from minutes $3-8$, whereas from minutes $5-8$, trained and control animals exhibited statistically similar freezing behavior. This observation adds further support to the assumption that the observed changes are a consequence of the associative CS-US learning, rather than a side effect of the expression of freezing.

CoA receives a fairly dense innervation from the olfactory bulb, and in turn, sends projections to the deep nuclei of the amygdala (Swanson and Petrovich 1998). Unlike other sensory systems, olfactory information does not reach the amygdala through a thalamic input. Instead, corticomedial amygdala (including $\mathrm{CoA}$ ) is the primary amygdaloid target of olfactory information (Price 1973; McDonald 1998). CoA sends projections to BLA and central nucleus (McDonald 1998), which have both been shown to receive nociceptive inputs (LeDoux 2000). Projections from the CoA to deep nuclei may therefore allow the CS-US association. Whether the amygdala is the final storage site of the association (LeDoux 2000), or allows the long-term consolidation of memory in other cortical sites (McGaugh 2002, 2004) is still a matter of debate. The lasting changes observed in CoA in the present study could suggest that this structure is involved in long-term storage of the association. The superficial nuclei of the amygdala (among which is CoA) have been shown to be a major source of the projections from the amygdala to the hypothalamus (Price et al. 1991), which is involved in the control of autonomic responses participating in fear behavior. Therefore, the increase in CoA following olfactory fear learning could favor the development of a rapid behavioral response in presence of the learned stressful odor.

\section{Conclusion}

The present study was the first to investigate the electrophysiological correlates of olfactory fear conditioning in behaving animals at several levels of the olfactory pathways. Our data suggest that the neural network involved in odor-shock association presents some particularities that could be linked to the uniqueness of the connections between the olfactory bulb and the amygdala. Hence, the cortical nucleus of the amygdala, which is part of the corticomedial amygdala that constitutes the primary amygdaloid target of olfactory information, could be involved in fear conditioning as far as an odor is used as the conditioned stimulus. A better knowledge of the neural circuit participating in this simple form of learning could help to understand the privileged connection between emotion and olfaction.

\section{Materials and Methods}

\section{Experiment 1}

\section{Subjects}

Twenty-four male Wistar rats (Charles River) weighing 250-300 g at the start of the experimentation served as subjects. They were housed individually, and food and water were available ad libitum. All animals were handled each day before the beginning of the experiments. Experiments were performed in accordance with the European guidelines regarding the care and use of animals for experimental procedures. 


\section{Behavioral apparatus}

\section{Conditioning cage and procedure}

The cage consisted of a Plexiglas transparent cylinder (diameter, $21 \mathrm{~cm}$; height, $21.5 \mathrm{~cm}$ ) with a lateral door (Vigouroux and Royet 1981), housed in a sound-attenuating enclosure. The floor of the cage consisted of 17 stainless-steel bars, $0.5 \mathrm{~cm}$ in diameter, that were spaced $1 \mathrm{~cm}$ apart. The floor was connected to a Coulbourn shock generator which, when appropriate, delivered $0.4 \mathrm{~mA}$ of scrambled shock. The ceiling of the cage was perforated with a central aperture (diameter, $2 \mathrm{~cm}$ ), which allowed the passage of recording cables when needed. In addition, the ceiling of the cage allowed the branching of three Tygon tubing connected to an olfactometer located outside of the apparatus. Deodorized air flowed constantly through the cage. At appropriate times, odor was introduced in the air stream for $10 \mathrm{sec}$. The conditioning cage was placed above a cubic Plexiglas chamber $(30 \times 30 \times 15$ $\mathrm{cm})$, on which an exhaust fan was mounted, allowing a continuous evacuation of the odorant stream from the conditioning cage. Using this paradigm, odor was perceived for $\sim 20 \mathrm{sec}$ at the experimenter nose. Two odorants have been used in this experiment, Isoamylacetate and Eugenol (Sigma).

Conditioning took place in a single session. The conditioning procedure was inspired from Otto et al (1997). Rats were introduced in the conditioning cage and given 2 min of free exploration. At the third minute, Isoamylacetate (conditioned stimulus, CS) was introduced in the cage for $20 \mathrm{sec}$, the last $2 \mathrm{sec}$ of which overlapped with the delivery of a $0.4-\mathrm{mA}$ foot-shock (unconditioned stimulus, US). The animals received six pairings of odor and shock with an intertrial interval of $4 \mathrm{~min}$. Twelve animals were trained as described above (trained group) and 12 animals received the same paradigm of odor presentation, but no shock was delivered (control group).

\section{CS-test session}

Assessment of CS-conditioned fear was performed $24 \mathrm{~h}$ after the conditioning session in a testing cage different from the conditioning cage, in order to avoid the influence of fear conditioned to the training context (Holland and Bouton 1999). The CStesting cage presented the same global characteristics as the conditioning cage, but the visual and tactile environmental cues have been changed; the walls of the cage were painted with vertical black and white stripes, and the floor was a thin metallic grid.

In both trained and control groups, two subgroups of rats were made according to the testing procedure (see Fig. 1, bottom, for a summary of the testing procedures). In one subgroup (Group 1, six trained animals, six control animals), rats were introduced in the testing cage and conditioned fear was assessed in an 8-min session. During the first 2 min of testing, no odorant was present, and the rats were free to explore their environment. Isoamylacetate (CS odor) was then presented during the first 20 sec of each of the following $6 \mathrm{~min}$. In the other subgroup (Group 2 , six trained animals, six control animals), the rats were submitted to two test sessions. The first test session was aimed at testing whether conditioned fear was specific to the conditioning odor, or could be evoked by a different odor. Therefore, the animals were tested as above, except that they were presented a novel odor, Eugenol, qualitatively different from the CS. During the second test session carried out $24 \mathrm{~h}$ later, the animals were tested for their response to the learned CS, Isoamylacetate.

During the different test sessions, animals' behavior was continuously monitored with a camera connected to a video recorder for off-line analysis.

\section{Context-test session}

Assessment of conditioned fear to contextual cues was performed $24 \mathrm{~h}$ after the CS-test. The animals were introduced in the conditioning cage and their freezing behavior was assessed over a 6-min period. The animals' behavior was continuously monitored with the video system for off-line analysis.

\section{Data analysis}

Off-line, animal behavior recorded during the test sessions was rated using an ethological keyboard connected to a home-made software (Matlab). Freezing behavior, characterized by a crouching posture and an absence of any visible movement except that due to breathing (Blanchard and Blanchard 1969) was quantified throughout the 8-min CS-test session and the 6-min context-test session. For each minute of the session, duration of freezing behavior was expressed as a percentage of the entire minute duration.

Percentages of freezing measured throughout the test sessions were compared using a two-factor (minute as a dependant factor and group as an independent factor) analysis of variance (ANOVA), followed by Wilcoxon matched-pairs signed-ranks tests for within group comparisons, and Mann-Witney tests for between groups' comparisons. For all of the statistical comparisons performed, the significance level was set at 0.05 .

\section{Experiment 2}

\section{Subjects and surgery}

Seventeen male Wistar rats (IFFA-CREDO) weighing 250-300 g at the start of the experimentation served as subjects. They were housed individually and food and water were available ad libitum. The animals were handled each day between their surgery and behavioral testing. Experiments were performed in accordance with the European guidelines regarding the care and use of animals for experimental procedures. They were anesthetized with Equithesin (a mixture of chloral hydrate and sodium pentobarbital; $3 \mathrm{ml} / \mathrm{kg}$, i.p.). The level of anesthesia was held constant with regular injections of Equithesin throughout surgery. The animals were fixed in a stereotaxic apparatus, with the head flat and holes were drilled for implantation of one bipolar stimulating electrode in the left olfactory bulb $(\mathrm{A} / \mathrm{P}-6 \mathrm{~mm}$ relative to the nasofrontal suture, L $1.3 \mathrm{~mm}$ relative to Bregma) and four monopolar recording electrodes positioned ipsilaterally in the aPC (A/P $+2.2 \mathrm{~mm}, \mathrm{~L} 4 \mathrm{~mm}$ relative to Bregma), the pPC (A/P $-1.8 \mathrm{~mm}$, L $5.5 \mathrm{~mm}$ to Bregma), the CoA (A/P $-2.3 \mathrm{~mm}$, L: 3.3 $\mathrm{mm}$ relative to Bregma), and the BLA (A/P $-2.8 \mathrm{~mm}$, L $4.9 \mathrm{~mm}$ relative to Bregma). The bipolar stimulating electrodes consisted of two $100-\mu \mathrm{m}$ stainless-steel wires (California Fine Wire) with a tip separation of $500 \mu \mathrm{m}$ in depth. The recording electrodes consisted of single 100- $\mu \mathrm{m}$ stainless steel wires. The depth of the stimulating electrodes was adjusted at the level of mitral cell layer using electrophysiological monitoring of the characteristic large multiunit mitral cell activity. Accurate positioning of recording electrodes' depth was achieved using the field-potential profile evoked in each structure in response to electrical stimulation of the bipolar $\mathrm{OB}$ electrodes (Fig. 4). In aPC, pPC, and CoA, recording electrode tips were positioned in the deep cortical layers (layer III), where the field potential signal presented a large stable amplitude, which corresponded to the approximate depths of $-7,-8$, and $-9 \mathrm{~mm}$, respectively. In BLA, the recording electrode tip was positioned at an approximate depth of $7.5 \mathrm{~mm}$. One skull screw placed above the contralateral parietal cortex served as a ground and reference electrode for monopolar field potential recordings. The recording and stimulating electrodes were connected to miniature sockets fixed onto the rat's head by dental cement. The animals were allowed 2 wk of post-surgical recovery.

\section{Stimulating and recording procedures}

Electrical stimulation used to induce evoked field potentials (EFPs) was delivered through a Master-8 stimulator (AMPI,) and a photically isolated constant current unit. The electrical stimulus was a single monophasic square pulse, $0.2 \mathrm{msec}$ in duration, 0.2 $\mathrm{Hz}$ in frequency. Stimulation intensity (200-400 $\mu \mathrm{A})$ was set to induce a response amplitude of $\sim 70 \%$ of maximum as determined from the baseline input-output curves. Each acquisition episode consisted of collecting 12 sweeps in parallel, through the four recording electrodes, in response to stimulation of the $\mathrm{OB}$ electrode. The signals induced in the four recording sites (Fig. 3B) were displayed on two oscilloscopes, amplified (Grass Model 12, Astro-Med, Inc.), filtered (1-300 Hz), and digitized (sampling frequency, $5 \mathrm{kHz}$ ) using a data acquisition system (Wavebook 512, Iotech, Inc.) for storage on a computer hard disk. 


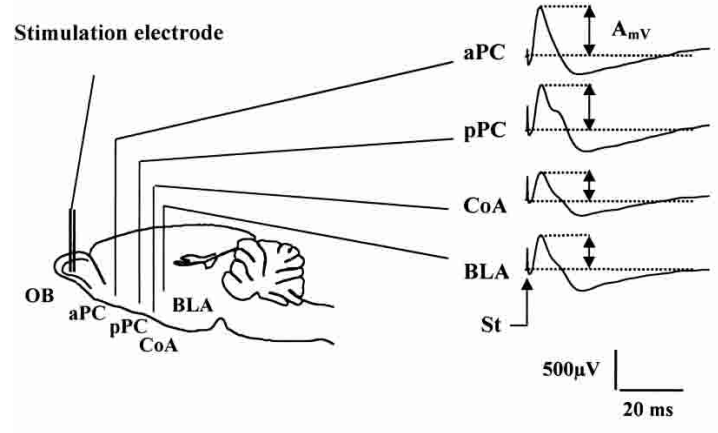

A

B
C

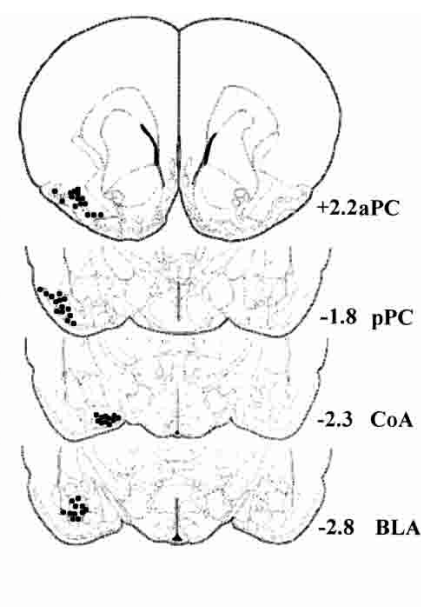

Figure 4. Schematic representation of the implanted electrodes and recorded evoked fieldpotential (EFPs) signals. (A) A bipolar stimulation electrode was implanted in the mitral cell layer of the olfactory bulb (OB). Four monopolar recording electrodes were respectively implanted in the anterior piriform cortex $(\mathrm{aPC})$, posterior piriform cortex (pPC), cortical nucleus of the amygdala (CoA), and basolateral amygdala (BLA). (B) An example of EFPs induced in the four recording sites in response to electrical stimulation of the OB. The amplitude $\left(A_{m v}\right)$ of the evoked main component was measured in each site, as indicated on the figure. (St) Stimulation artifact. (C) Schematic drawing representing recording electrodes' tip placements. Numbers on the right indicate position of the coronal sections in millimeter relative to bregma (Paxinos and Watson 1998).

\section{Procedure}

\section{Baseline recording}

Baseline recording of field potentials was established over a 4-d period, with one recording session per day. The rat was placed in the testing cage (see Materials and Methods, Experiment 1). Recording and stimulating cables were relayed at the top of the cage through a multichannel swiveling electrical connector. The recording cable contained a 5-channel JFET headstage in order to reduce movement artifacts.

The first two sessions were aimed at establishing inputoutput curves. During these sessions, stimulation intensity was varied from 100 to $700 \mu \mathrm{A}$. For each rat, the intensity inducing a response around $70 \%$ of the maximum was determined and used for the subsequent recording sessions. The two last sessions were aimed at collecting baseline preconditioning signals.

\section{Conditioning}

Twenty-four hours after the last baseline recording session, the rats were assigned to two groups as follows: the trained group $(n=9)$ and the control group $(n=8)$. In the trained group, the rats were placed in the conditioning cage and received six pairings of Isoamylacetate and foot-shock as described above (see Materials and Methods, Experiment 1). In the control group, the rats received the same number of Isoamylacetate presentations, but no foot-shock was delivered. No recording was carried out during the conditioning session.

\section{CS-test session}

Twenty-four hours after the conditioning session, the animals of both groups were introduced in the CS-testing cage (see Materials and Methods, Experiment 1), connected to the recording apparatus, and tested for their behavioral response to the repeated presentation of Isoamylacetate as described above. In parallel, during the 8-min duration of the session, EFP signals were continuously collected in the four recording sites.

\section{Context-test session}

Twenty-four hours after the CS-test session, the animals of both groups were introduced in the conditioning cage (see Materials and Methods, Experiment 1) and tested for their behavioral response to contextual cues.

\section{Data analysis}

Behavioral data

Percentages of freezing were measured throughout the CS and context test sessions as described in Experiment 1, and compared using a two-factor (minute as a dependant factor and group as an independent factor) analysis of variance (ANOVA), followed by Wilcoxon matched-pairs, signed-ranks tests for within group comparisons, and Mann-Whitney tests for between groups' comparisons. For all of the statistical comparisons performed, the significance level was set at 0.05 .

\section{Electrophysiological data}

EFPs were analyzed using the data acquisition software Dasylab (Iotech, Inc.). Offline, the individual traces collected during each baseline session (12 sweeps) were averaged, thus resulting in a mean baseline signal for each recording site. During the 8-min CS-test session, two kinds of analyses were carried out. First, the individual traces collected during each minute (12 sweeps) of the test session were averaged per minute, which led to eight mean EFPs (one per minute) for each recording site. Second, the mean signals obtained for the first $2 \mathrm{~min}$ (pre-odor period) and the mean signals obtained for the last $6 \mathrm{~min}$ (odor-period) were pooled separately. In each recording site, the peak amplitude of the main positive component of the mean EFP was measured as illustrated in Figure 4B. The peak amplitudes obtained during the CS-test session were expressed as percentages of the baseline values collected before learning. For each recording site, the data were compared using a two-factor (group as an independent factor and minute as a dependant factor) analysis of variance (ANOVA), followed by Wilcoxon matched-pairs, signed-ranks tests for within group comparisons, and Mann-Whitney tests for between groups' comparisons. For all of the statistical comparisons performed, the significance level was set at 0.05.

\section{Histology}

At the end of the experiment, rats were injected with a lethal dose of pentobarbital. Brains were dissected and stored in a $10 \%$ formalin solution for $1 \mathrm{wk}$, after which brains were cut into 80$\mu \mathrm{m}$ slices and stained with cresyl violet. For each rat, the position of each recording electrode was determined using Paxinos and Watson's (1998) stereotaxic atlas (Fig. 4C).

\section{Acknowledgments}

We gratefully acknowledge Jean-Luc Charieau for taking care of the animals and Hassen Slimani for technical support, as well as Lionel Augeul for his help during the pilot behavioral experiments. We also warmly thank Dr. Regina Sullivan and Tania Roth for a careful review of the English.

\section{References}

Baldi, E., Lorenzini, C.A., and Bucherelli, C. 2004. Footshock intensity and generalization in contextual and auditory-cued fear conditioning in the rat. Neurobiol. Learn. Mem. 81: 162-166.

Blanchard, R.J. and Blanchard, D.C. 1969. Crouching as an index of fear. J. Comp. Physiol. Psychol. 67: 370-375.

Bouret, S. and Sara, S.J. 2002. Locus coeruleus activation modulates firing rate and temporal organization of odor-induced single-cell responses in rat piriform cortex. Eur. J. Neurosci. 16: 2371-2382.

Chabaud, P., Ravel, N., Wilson, D.A., and Gervais, R. 1999. Functional coupling in rat central olfactory pathways: A coherence analysis. Neurosci. Lett. 276: 17-20.

Chabaud, P., Ravel, N., Wilson, D.A., Mouly, A.M., Vigouroux, M., Farget, V., and Gervais, R. 2000. Exposure to behaviorally relevant 
odor reveals differential characteristics in rat central olfactory pathways as studied through oscillatory activities. Chem. Senses 25: $561-573$.

Cousens, G. and Otto, T. 1998. Both pre- and post-training excitotoxic lesions of the basolateral amygdala abolish the expression of olfactory and contextual fear conditioning. Behav. Neurosci.

112: 1092-1103.

Datiche, F. and Cattarelli, M. 1996. Catecholamine innervation of the piriform cortex: A tracing and immunohistochemical study in the rat. Brain. Res. 710: $69-78$.

Desmedt, A., Garcia, R., and Jaffard, R. 2003. An 8-day extensive elemental, but not contextual, fear conditioning potentiates hippocampal-lateral septal synaptic efficacy in mice. Synapse 49: 270-278.

Doyère, V., Redini-Del Negro, C., Dutrieux, G., Le Floch, G., Davis, S., and Laroche, S. 1995. Potentiation or depression of synaptic efficacy in the dentate gyrus is determined by the relationship between the conditioned and unconditioned stimulus in a classical conditioning paradigm in rats. Behav. Brain. Res. 70: 15-29.

Funk, D. and Amir, S. 2000. Enhanced fos expression within the primary olfactory and limbic pathways induced by an aversive conditioned odor stimulus. Neuroscience 98: 403-406.

Goosens, K.A., Hobin, J.A., and Maren, S. 2003. Auditory-evoked spike firing in the lateral amygdala and Pavlovian fear conditioning: Mnemonic code or fear bias? Neuron 40: 1013-1022.

Haberly, L.B. 1973. Summed potentials evoked in opossum prepyriform cortex. J. Neurophysiol. 36: 775-788.

2001. Parallel-distributed processing in olfactory cortex: New insights from morphological and physiological analysis of neuronal circuitry. Chem. Senses 26: 551-576.

Hasselmo, M.E., Linster, C., Patil, M., Ma, D., and Cekic, M. 1997. Noradrenergic suppression of synaptic transmission may influence cortical signal-to-noise ratio. J. Neurophysiol. 77: 3326-3339.

Herry, C., Vouimba, R.M., and Garcia, R. 1999. Plasticity in the mediodorsal thalamo-prefrontal cortical transmission in behaving mice. J. Neurophysiol. 82: 2827-2832.

Herzog, C. and Otto, T. 1997. Odor-guided fear conditioning in rats: 2. Lesions of the anterior perirhinal cortex disrupt fear conditioned to the explicit conditioned stimulus but not to the training context. Behav. Neurosci. 111: 1265-1272.

Holland, P.C. and Bouton, M.E. 1999. Hippocampus and context in classical conditioning. Curr. Opin. Neurobiol. 9: 195-202.

Ketchum, K.L. and Haberly, L.B. 1993. Membrane currents evoked by afferent fiber stimulation in rat piriform cortex. I. Current source-density analysis. J. Neurophysiol. 69: 248-260.

Kilpatrick, L. and Cahill, L. 2003. Modulation of memory consolidation for olfactory learning by reversible inactivation of the basolateral amygdala. Behav. Neurosci. 117: 184-188.

Krettek, J.E. and Price, J.L. 1978. A description of the amygdaloid complex in the rat and cat with observations on intra-amygdaloid axonal connections. J. Comp. Neurol. 178: 255-280.

LeDoux, J.E. 2000. Emotion circuits in the brain. Annu. Rev. Neurosci. 23: $155-184$.

Litaudon, P., Mouly, A.M., Sullivan, R., Gervais, R., and Cattarelli, M. 1997. Learning-induced changes in rat piriform cortex activity mapped using multisite recording with voltage sensitive dye. Eur. J. Neurosci. 9: 1593-1602.

Litaudon, P., Amat, C., Bertrand, B., Vigouroux, M., and Buonviso, N. 2003. Piriform cortex functional heterogeneity revealed by cellular responses to odours. Eur. J. Neurosci. 17: 2457-2461.

Luskin, M.B. and Price, J.L. 1983. The topographic organization of associational fibers of the olfactory system in the rat, including centrifugal fibers to the olfactory bulb. J. Comp. Neurol. 216: 264-291.

Maren, S. 2001. Neurobiology of Pavlovian fear conditioning. Annu. Rev. Neurosci. 24: 897-931.

Martin, S.J. and Morris, R.G. 2002. New life in an old idea: The synaptic plasticity and memory hypothesis revisited. Hippocampus 12: 609-636.

McDonald, A.J. 1998. Cortical pathways to the mammalian amygdala. Prog. Neurobiol. 55: 257-332.

McGaugh, J.L. 2002. Memory consolidation and the amygdala: A systems perspective. Trends Neurosci. 25: 456.

. 2004. The amygdala modulates the consolidation of memories of emotionally arousing experiences. Annu. Rev. Neurosci. 27: 1-28.

McGaugh, J.L. and Roozendaal, B. 2002. Role of adrenal stress hormones in forming lasting memories in the brain. Curr. Opin. Neurobiol. 12: $205-210$.

McNamara, A.M., Cleland, T.A., and Linster, C. 2004. Characterization of the synaptic properties of olfactory bulb projections. Chem. Senses 29: $225-233$

Moriceau, S., Roth, T.L., Okotoghaide, T., and Sullivan, R.M. 2004. Corticosterone controls the developmental emergence of fear and amygadala function to predator odors in infant rat pups. Int. J. Dev. Neurosci. 22: 415-422.
Moser, E., Mathiesen, I., and Andersen, P. 1993. Association between brain temperature and dentate field potentials in exploring and swimming rats. Science 259: 1324-1326.

Mouly, A.M., and Gervais, R. 2002. Polysynaptic potentiation at different levels of rat olfactory pathways following learning. Learn. Mem. 9: 66-75.

Mouly, A.M., Fort, A., Ben-Boutayab, N., and Gervais, R. 2001. Olfactory learning induces differential long-lasting changes in rat central olfactory pathways. Neuroscience 102: 11-21.

Ottersen, O.P. 1982. Connections of the amygdala of the rat. IV: Corticoamygdaloid and intraamygdaloid connections as studied with axonal transport of horseradish peroxidase. J. Comp. Neurol. 205: $30-48$.

Otto, T., Cousens, G., and Rajewski, K. 1997. Odor-guided fear conditioning in rats: 1 . Acquisition, retention, and latent inhibition. Behav. Neurosci. 111: 1257-1264.

Otto, T., Cousens, G., and Herzog, C. 2000. Behavioral and neuropsychological foundations of olfactory fear conditioning. Behav. Brain Res. 110: 119-128.

Paschall, G.Y. and Davis, M. 2002. Olfactory-mediated fear-potentiated startle. Behav. Neurosci. 116: 4-12.

Paxinos, G. and Watson, C. 1998. The rat brain in stereotaxic coordinates. Fourth edition, Academic Press, San Diego, CA.

Price, J.L. 1973. An autoradiographic study of complementary laminar patterns of termination of afferent fibers to the olfactory cortex. $J$. Comp. Neurol. 150: 87-108.

Price, J.L., Slotnick, B.M., and Revial, M.F. 1991. Olfactory projections to the hypothalamus. I. Comp. Neurol. 306: 447-461.

Richardson, R., Vishney, A., and Lee, J. 1999. Conditioned odor potentiation of startle in rats. Behav. Neurosci. 113: 787-794.

Richardson, R., Tronson, N., Bailey, G.K., and Parnas, A.S. 2002. Extinction of conditioned odor potentiation of startle. Neurobiol. Learn. Mem. 78: 426-440.

Rogan, M.T., Staubli, U.V., and LeDoux, J.E. 1997. Fear conditioning induces associative long-term potentiation in the amygdala. Nature 390: 604-607.

Rosenkranz, J.A. and Grace, A.A. 2002. Dopamine-mediated modulation of odour-evoked amygdala potentials during pavlovian conditioning. Nature 417: 282-287.

Sara, S.J. and Segal, M. 1991. Plasticity of sensory responses of locus coeruleus neurons in the behaving rat: Implications for cognition. Prog. Brain. Res. 88: 571-585.

Savander, V., LeDoux, J.E., and Pitkanen, A. 1996. Topographic projections from the periamygdaloid cortex to select subregions of the lateral nucleus of the amygdala in the rat. Neurosci. Lett. 211: 167-170.

Schettino, L.F. and Otto, T. 2001. Patterns of Fos expression in the amygdala and ventral perirhinal cortex induced by training in an olfactory fear conditioning paradigm. Behav. Neurosci. 115: 1257-1272.

Slotnick, B. 2001. Animal cognition and the rat olfactory system. Trends Cogn. Sci. 5: 216-222.

Sullivan, R.M. 2001. Unique characteristics of neonatal classical conditioning: The role of the amygdala and locus coeruleus. Int. Physiol. Behav. Sci. 36: 293-307.

Sullivan, R.M., Landers, M., Yeaman, B., and Wilson, D.A. 2000. Good memories of bad events in infancy. Nature 407: 38-39.

Swanson, L.W. and Petrovich, G.D. 1998. What is the amygdala? Trends Neurosci. 21: 323-331.

Tang, J., Wotjak, C.T., Wagner, S., Williams, G., Schachner, M., and Dityatev, A. 2001. Potentiated amygdaloid auditory-evoked potentials and freezing behavior after fear conditioning in mice. Brain Res. 919: 232-241.

Tang, J., Wagner, S., Schachner, M., Dityatev, A., and Wotjak, C.T. 2003. Potentiation of amygdaloid and hippocampal auditory-evoked potentials in a discriminatory fear-conditioning task in mice as a function of tone pattern and context. Eur. J. Neurosci. 18: 639-650.

Vigouroux, M. and Royet, J.P. 1981. An olfactometric cage suitable for short duration stimulations of unrestrained small animals. $J$. Neurosci. Methods 4: 189-196.

Wilson, D.A. 1998a. Synaptic correlates of odor habituation in the rat anterior piriform cortex. J. Neurophysiol. 80: 998-1001.

- 1998b. Habituation of odor responses in the rat anterior piriform cortex. J. Neurophysiol 79: 1425-1440.

. 2000. Comparison of odor receptive field plasticity in the rat olfactory bulb and anterior piriform cortex. J. Neurophysiol. 84: $3036-3042$.

2003. Rapid, experience-induced enhancement in odorant discrimination by anterior piriform cortex neurons. J. Neurophysiol. 90: $65-72$.

Received July 13, 2004; accepted in revised form October 5, 2004. 


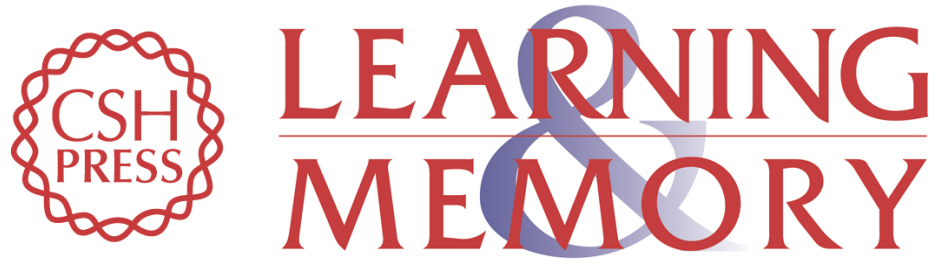

\section{Olfactory fear conditioning induces field potential potentiation in rat olfactory cortex and amygdala}

Yannick Sevelinges, Rémi Gervais, Belkacem Messaoudi, et al.

Learn. Mem. 2004, 11:

Access the most recent version at doi:10.1101/lm.83604

References This article cites 58 articles, 2 of which can be accessed free at:

http://learnmem.cshlp.org/content/11/6/761.full.html\#ref-list-1

License

Email Alerting Receive free email alerts when new articles cite this article - sign up in the box at the Service top right corner of the article or click here. 